In Cres. Vol. $4 N^{\circ} 1:$ pp. 121-131, 2013

\title{
SATISFACCIÓN LABORAL Y CALIDAD DE VIDA PROFESIONAL DE LAS ENFERMERAS DEL HOSPITAL REGIONAL DOCENTE DE TRUJILLO. 2009*
}

\author{
LABOUR SATISFACTION AND LIFE QUALITY OF NURSES OF THE \\ REGIONAL TEACHING HOSPITAL OF TRUJILLO, 2009.
}

\author{
Asunción Marcela Urbina Baltodano ${ }^{1}$, \\ Flor Marlene Luna Victoria Mori ${ }^{2}$
}

\begin{abstract}
RESUMEN
La presente investigación se realizó con el objetivo de determinar la relación del nivel de satisfacción laboral y nivel de calidad de vida profesional de las enfermeras del Hospital Regional Docente de Trujillo, con enfoque teórico conductista, basado en Herzberg, Maslow. El diseño de investigación fue descriptivo correlacional; el universo muestral estuvo constituido por 84 enfermeras, a quienes se aplicó dos instrumentos: la escala de satisfacción laboral de Núñez y calidad de vida profesional CVP 35 Sánchez. El análisis de los datos se realizó con el paquete estadístico SPSS versión 18.0 y la prueba estadística $\mathrm{X}^{2}$. Las dimensiones con un nivel alto de satisfacción laboral son las relacionadas con la competencia profesional y la propia satisfacción del trabajo; y con un nivel bajo, las de promoción profesional, presión en el trabajo. Las dimensiones con un nivel bueno de calidad de vida profesional son la motivación intrínseca y la capacidad de realizar el trabajo y la de bajo nivel, la carga de trabajo percibido. Existe una relación significativa entre la satisfacción laboral y la calidad de vida profesional.
\end{abstract}

PALABRAS CLAVE: Calidad de vida profesional, satisfacción laboral.

* Recibido: 24 de abril del 2013; aprobado: 30 de mayo del 2013.

1 Magister en Enfermería, Mención: Salud del Adulto. Doctoranda en Ciencias en Enfermería. Profesora Principal del Departamento de Enfermería del Adulto y Anciano. UNT- Trujillo.

2 Doctora en Enfermería, Docente de Postgrado UNT. Profesora Principal del Departamento de Enfermería del Adulto y Anciano. UNT- Trujillo. 


\begin{abstract}
This non-experimental research of descriptive correlational design aimed to establish the relationship between level of labour satisfaction and quality of professional life in nurses of the Regional Teaching Hospital of Trujillo (Peru), with the behavioral theoretical approach, based on Herzberg-Maslow. The sample consisted of 84nurses. The instruments used were the labour satisfaction and professional quality of life scale (CVP). The data was statistically analyzed by the Statistical Package for Social Science (SPSS) and the means of the Chi Square test. The study came to the following conclusions: The dimensions with a high level of labour satisfaction is the one related to the professional competence and job satisfaction and the low levels are related to the career development, work pressure. The dimensions with a good level of working life quality are intrinsic motivation and the ability to perform the work; the one of low level of satisfaction is the perceived workload. There is a significant relationship between labour satisfaction and quality of professional life.
\end{abstract}

KEY WORDS: Quality of professional life, labour satisfaction.

\title{
I. INTRODUCCIÓN
}

La globalización económica, social y cultural exigen al ser humano un desarrollo personal y profesional, considerando al trabajo como una actividad de vastos alcances que refleja e influye sobre casi todos los aspectos de la conducta humana; por ende, al desempeñar un trabajo no sólo trasladan a él sus habilidades intelectuales y motrices, sino también las individualidades. ${ }^{1}$

El trabajo no sólo desarrolla a la persona sino también a la profesión u oficio. Es así, como el profesional de enfermería, a lo largo de los años, ha ido desarrollando su modelo de cuidado como ciencia y profesión, con el objeto de satisfacer las necesidades de la sociedad cambiante; por ello, ser enfermera implica tener cualidades muy especiales como análisis crítico, creatividad, sensibilidad, y otros, para adaptar los servicios de salud a un entorno favorecedor para el cuidado del ser humano. ${ }^{2}$

Sin embargo, en la actualidad, la enfermera asistencial está sometida a una sobrecarga laboral, debido a que los pacientes, cada vez, son más exigentes y críticos; así mismo, existen múltiples factores que obligan a que, con frecuencia, se exija mayor rendimiento al profesional de enfermería, sin que nunca o casi nunca, se piense en su calidad de vida profesional.

No obstante, el ambiente donde se desarrollan el profesional de enfermería pareciera no ser el más propicio para derivar una satisfacción laboral; el cansan- 
cio, las escasas posibilidades de capacitación debido al sistema de turnos y la escasez de personal, traen como consecuencia fatiga y desmoralización entre las profesionales, lo que impide en algunas ocasiones concretar la esencia fundamental, el cuidado del usuario externo

Según Malvárez ${ }^{3}$, el personal de enfermería representa el 50 a $60 \%$ de la fuerza laboral del sistema de salud, por ser uno de los capitales humanos más importantes en salud; no sólo en el desempeño profesional, sino también por su capacidad resolutiva al conocer las necesidades reales o sentidas del usuario interno o externo, por lo que concentra el mayor porcentaje de costos fijos en los presupuestos de salud y participa, activamente, en la entrega de cuidados directos e indirectos a la persona, familias y a la comunidad; por tanto, constituye un importante grupo laboral.

Sin embargo, tal relevancia es antagónica, a que sólo el 37\% de las enfermeras a nivel mundial se encuentran satisfechas desde el punto de vista laboral de su trabajo, en sí, y a oportunidades de perfeccionamiento y menos satisfechas con los ascensos y las remuneraciones. En los países latinoamericanos, la insatisfacción laboral está dada principalmente por una baja remuneración económica. La identificación con la profesión y las relaciones profesionales son los aspectos que proporcionan más satisfacción laboral. ${ }^{4}$

En el Perú, las normas que rigen la profesión de enfermería parecieran no ser las más óptimas, debido a que no satisfacen las necesidades básicas, sumándose a esto, un desconocimiento del amparo legal dado por la ley del trabajo de la enfermera. Tal situación obliga al profesional de enfermería a migrar a otros países en busca de mejores oportunidades. En su mayoría, según el Ministerio de Salud, ${ }^{4}$ un 60,9\% emigra a EEUU, Italia y España.

En los últimos años, existe un gran interés entre los administradores y el gobierno por mejorar la calidad de vida en el trabajo, de ahí la necesidad de estudiar al trabajador en relación con su medio ambiente laboral, en el cual está inserto, ya que cualquier variación en el conjunto integrado por el hombre, la organización y el medio ambiente, afectará forzosamente a los demás. Por lo que se debe investigar, la satisfacción laboral y su calidad de vida laboral para conocer lo que ocurre a diario dentro de la organización de salud.

El presente estudio se sustenta en el conductismo, una de las corrientes filosóficas que más han influido en el área de trabajo, satisfacción laboral y, por tanto, en la calidad de vida profesional. La satisfacción laboral se aborda a tra- 
vés de la teoría de Bifactorial de Herzberg ${ }^{5}$ y la calidad de vida profesional, en la teoría de necesidades de Maslow ${ }^{6}$.

Herzberg $^{5}$ señala que existen dos tipos de factores motivacionales: un primer grupo, extrínseco al trabajo mismo, denominado "de higiene o mantención” y, un segundo grupo, intrínseco al trabajo, denominado "motivador".

Los factores higiénicos son las condiciones que rodean a la persona cuando desempeña un trabajo, están fuera del control de la persona, y cuando son óptimos, simplemente, evitan la insatisfacción y cuando son pésimos producen insatisfacción. Su efecto es como un medicamento para el dolor de cabeza, combaten el dolor pero no mejora la salud. Por estar relacionados con la insatisfacción, Herzberg ${ }^{5}$ los llama factores de insatisfacción.

Los factores motivacionales están bajo el control del individuo, se relacionan con lo que él hace y desempeña, involucrando sentimientos de crecimiento individual, de reconocimiento profesional; sin embargo, las tareas y cargos son diseñados para atender a los principios de eficiencia y de economía, suspendiendo oportunidades de creatividad de las personas. Esto hace perder el significado psicológico del individuo, lo que ocasiona un desinterés, provocando desmotivación, ya que la empresa sólo ofrece un lugar decente para trabajar. Según Herzberg, los factores motivacionales sobre el comportamiento de las personas son mucho más profundos y estables cuando son óptimos, elevan la satisfacción de modo sustancial y, cuando son pésimos, provocan pérdida de satisfacción, Por el hecho de estar ligados a la satisfacción del individuo Herzberg los llama factores de satisfacción.

Para objeto de estudio, la calidad de vida profesional está analizada a través de la teoría de Maslow ${ }^{6}$, "Teoría de las necesidades”, en la que señala que a medida que el hombre satisface sus necesidades básicas o primarias, otras más elevadas como las secundarias ocupan el predominio de su comportamiento y se vuelven imprescindibles, como es la calidad de vida profesional, debido a que el profesional de enfermería ha suplido sus necesidades primarias. Ante esto, plantea que el ser humano está constituido por un cuerpo físico, sociológico y espiritual y cualquier repercusión o problema que ocurre en cualquiera de estos cuerpos repercute automáticamente sobre el resto de los cuerpos, lo que podría estar dado si la enfermera tiene una insatisfacción laboral, lo que puede conllevar a una mala calidad de vida profesional.

Es por ello que la investigación describe que durante el transcurso de vida 
laboral, ha observado en distintos centros asistenciales los problemas y dificultades a los que se enfrentan las enfermeras asistenciales, en especial en la ciudad de Trujillo. Esta situación podría indicar que el nivel de satisfacción laboral de las enfermeras no es más propicio, debido a que existen conflictos que se generan dentro de los equipos de trabajo, dificultades con sus jefes directos, con sus pares y con el personal a su cargo, produciéndose un entorpecimiento en la comunicación; por tanto, se altera la armonía y el desarrollo del trabajo, tornándose dificultoso y poco gratificante.

\section{PROBLEMA}

¿Existe relación entre el nivel satisfacción laboral y el nivel de calidad de vida profesional de las enfermeras del área de hospitalización del Hospital Regional Docente de Trujillo, 2009?

\section{OBJETIVO GENERAL}

Determinar la relación entre el nivel de satisfacción laboral y el nivel de calidad de vida profesional de las enfermeras del área de hospitalización del Hospital Regional Docente de Trujillo, 2009.

\section{OBJETIVOS ESPECÍFICOS}

1. Determinar los niveles según dimensiones de la satisfacción laboral en las enfermeras que laboran en el área de hospitalización del Hospital Regional Docente de Trujillo, 2009.

2. Determinar los niveles según dimensiones de la calidad de vida profesional de las enfermeras que laboran en el área de hospitalización del Hospital Regional Docente de Trujillo, 2009.

\section{HIPÓTESIS}

Hi: A mayor nivel de satisfacción laboral, mayor nivel de la calidad de vida profesional de las enfermeras que laboran en el área de hospitalización del Hospital Regional Docente de Trujillo, 2009.

\section{MATERIAL Y MÉTODOS}

El presente estudio corresponde a un enfoque cuantitativo, de tipo no experimental, con diseño descriptivo-correlacional. El universo muestral, estuvo constituido por 84 enfermeras que laboran en el área de hospitalización del Hospital Regional Docente de Trujillo de Enero - Abril del 2009. 
En el presente estudio de investigación se utilizó 2 instrumentos:

Cuestionario de satisfacción laboral. ${ }^{7}$ De 26 ítems, agrupados en 9 dimensiones (satisfacción en el trabajo, tensión relacionada con el trabajo, competencia profesional, presión de trabajo, promoción profesional, relación interpersonal con el jefe, relación interpersonal con los compañeros, características extrínsecas de estatus, monotonía laboral).

Se categorizó:

Alto nivel de satisfacción laboral : 104 a 130 puntos.

Moderado nivel satisfacción laboral: 78 a 103 puntos.

Bajo nivel de satisfacción laboral : 26 a 77 puntos.

Cuestionario de calidad de vida CVP-35. ${ }^{8}$ De 35 preguntas cerradas, considerando 7 dimensiones (Incomodidad derivadas del trabajo, soporte emocional por parte de los directivos, carga de trabajo percibida, recursos ligados al lugar de trabajo, soporte social recibido, motivación intrínseca, capacidad de realizar el trabajo). Se categorizó en los siguientes niveles:

Nivel bueno de calidad de vida profesional : 246 a 350 .

Nivel regular de calidad de vida profesional : 140 a 245 .

Nivel malo de calidad de vida profesional : 35 a 139.

Para la ejecución se solicitó la autorización del Director del Hospital Regional Docente de Trujillo. Se coordinó con la enfermera jefa del servicio de enfermería. Se seleccionó el universo muestral de acuerdo a los criterios de inclusión. Se aplicó los instrumentos a cada enfermera, explicándose los objetivos del estudio mediante una encuesta en un tiempo máximo de 20 minutos. El procesamiento y análisis estadístico se realizó mediante la clasificación, ordenamiento y codificación de datos mediante el programa SPSS/info/software, versión 18.0, utilizándose la prueba estadística $X^{2}$. Para el análisis inferencial, se tuvo en cuenta los principios éticos: autonomía, beneficencia, no maleficencia, justicia. 


\section{RESULTADOS}

Tabla 01

NIVEL DE LAS DIMENSIONES DE SATISFACCIÓN LABORAL EN ENFERMERAS. HOSPITAL REGIONAL DOCENTE DE TRUJILLO. 2009

\begin{tabular}{|c|c|c|c|c|c|c|c|c|}
\hline \multirow{2}{*}{$\begin{array}{c}\text { Dimensiones de satisfacción } \\
\text { Laboral }\end{array}$} & \multicolumn{2}{|c|}{ Alto } & \multicolumn{2}{|c|}{ Moderado } & \multicolumn{2}{|c|}{ Bajo } & \multicolumn{2}{|c|}{ Total } \\
\hline & f & $\%$ & $\mathbf{f}$ & $\%$ & f & $\%$ & f & $\%$ \\
\hline Satisfacción en el trabajo. & 18 & 21,0 & 58 & 69,0 & 8 & 10,0 & 84 & 100,0 \\
\hline Tensión relacionada con trabajo. & 0 & 0,0 & 60 & 71,0 & 24 & 29,0 & 84 & 100,0 \\
\hline Competencia profesional. & 40 & 48,0 & 44 & 52,0 & 0 & 0,0 & 84 & 100,0 \\
\hline Presión de trabajo. & 0 & 0,0 & 45 & 54,0 & 39 & 46,0 & 84 & 100,0 \\
\hline Promoción profesional. & 0 & 0,0 & 33 & 40,0 & 51 & 60,0 & 84 & 100,0 \\
\hline $\begin{array}{l}\text { Relación interpersonal con el } \\
\text { jefe. }\end{array}$ & 10 & 12,0 & 64 & 76,0 & 10 & 12,0 & 84 & 100,0 \\
\hline $\begin{array}{l}\text { Relación interpersonal con los } \\
\text { compañeros. }\end{array}$ & 16 & 19,0 & 45 & 54,0 & 23 & 27,0 & 84 & 100,0 \\
\hline $\begin{array}{l}\text { Características extrínsecas de } \\
\text { status. }\end{array}$ & 3 & 4,0 & 66 & 79,0 & 15 & 17,0 & 84 & 100,0 \\
\hline Monotonía laboral. & 0 & 0,0 & 61 & 73,0 & 23 & 27,0 & 84 & 100,0 \\
\hline
\end{tabular}

Fuente: Cuestionario de Satisfacción laboral de Núñez, aplicado a las enfermeras asistenciales. HRDT.

Tabla 02

NIVEL DE LAS DIMENSIONES DE LA CALIDAD DE VIDA PROFESIONAL EN LAS ENFERMERAS. HOSPITAL REGIONAL DOCENTE DE TRUJILLO. 2009

\begin{tabular}{lcccccccc}
\hline \begin{tabular}{l} 
Dimensiones de calidad de \\
\multicolumn{1}{c}{ vida profesional }
\end{tabular} & \multicolumn{2}{c}{ Bueno } & \multicolumn{2}{c}{ Regular } & \multicolumn{2}{c}{ Mala } & \multicolumn{2}{c}{ Total } \\
\hline $\begin{array}{l}\text { Incomodidad derivadas del } \\
\text { trabajo. }\end{array}$ & 0 & 0,0 & 55 & 65,0 & 29 & 35,0 & 84 & 100,0 \\
$\begin{array}{l}\text { Soporte emocional por parte } \\
\text { de los directivos. }\end{array}$ & 0 & 0,0 & 54 & 64,0 & 30 & 36,0 & 84 & 100,0 \\
Carga de trabajo percibida. & 0 & 0,0 & 39 & 46,0 & 45 & 54,0 & 84 & 100,0 \\
$\begin{array}{l}\text { Recursos ligados al lugar de } \\
\text { trabajo. }\end{array}$ & 0 & 0,0 & 61 & 73,0 & 23 & 27,0 & 84 & 100,0 \\
$\begin{array}{l}\text { Soporte social recibido. } \\
\text { Motivación intrínseca. }\end{array}$ & 0 & 0,0 & 54 & 64,0 & 30 & 36,0 & 84 & 100,0 \\
$\begin{array}{l}\text { Capacidad de realizar el } \\
\text { trabajo. }\end{array}$ & 8 & 9,5 & 79 & 94,0 & 5 & 6,0 & 84 & 100,0 \\
\hline
\end{tabular}

Fuente: Cuestionario de calidad de vida profesional CVP 35 Sánchez aplicado a las enfermeras asistenciales HRDT. 
Tabla 03

NIVEL DE SATISFACCIÓN LABORAL Y SU RELACIÓN CON EL NIVEL DE CALIDAD DE VIDA PROFESIONAL EN LAS ENFERMERAS. HRDT. 2009.

\begin{tabular}{|c|c|c|c|c|c|c|c|c|}
\hline \multirow{3}{*}{$\begin{array}{l}\text { Calidad de } \\
\text { vida }\end{array}$} & \multicolumn{6}{|c|}{ Satisfacción laboral } & \multirow{2}{*}{\multicolumn{2}{|c|}{ Total }} \\
\hline & \multicolumn{2}{|c|}{ Alto } & \multicolumn{2}{|c|}{ Moderado } & \multicolumn{2}{|c|}{ Bajo } & & \\
\hline & f & $\%$ & $\mathbf{f}$ & $\%$ & f & $\%$ & $\mathbf{f}$ & $\%$ \\
\hline Bueno & 4 & 40,0 & 11 & 21,2 & 0 & 0,0 & 15 & 19,7 \\
\hline Regular & 6 & 60,0 & 39 & 75,0 & 18 & 81,8 & 63 & 75,0 \\
\hline Mala & 0 & 0,0 & 2 & 3,8 & 4 & 18,2 & 6 & 7,1 \\
\hline Total & 10 & 100,0 & 52 & 100,0 & 22 & 100,0 & 84 & 100,0 \\
\hline
\end{tabular}

$\mathrm{X}^{2}=12,686 \quad \mathrm{gl}=4 \quad \mathrm{p}=0,013<0,05$ Significativo

Fuente: Cuestionario del nivel de satisfacción laboral y de nivel de calidad de vida profesional aplicado a las enfermeras asistenciales HRDT.

En la tabla 01, se evidencia que las dimensiones con alta satisfacción laboral en las enfermeras del Hospital Regional Docente de Trujillo (HRDT) son las relacionadas con la competencia profesional $(48,0 \%)$ y la propia satisfacción del trabajo (21,0\%); y las enfermeras con un nivel bajo en las dimensiones de la satisfacción laboral son la relacionadas con promoción profesional $(60,0 \%)$, presión en el trabajo (46\%), tensión relacionada con el trabajo $(29,0 \%)$.

En la tabla 02 se evidencia que las dimensiones con un nivel bueno en la calidad de vida profesional son representadas en un $14,3 \%$ y $9,5 \%$ en las dimensiones capacidad de realizar el trabajo y motivación intrínseca, respectivamente; situación diferente al $54,0 \%$ de enfermeras que presenta un nivel malo en dimensión carga de trabajo percibido, seguido del 36,0\% de nivel malo en la dimensión soporte emocional por parte de los directivos, al igual que el $36,0 \%$ de soporte social recibido.

En la tabla 03, se evidencia que las enfermeras con un nivel alto de satisfacción laboral presentan una calidad de vida profesional de regular $(60,0 \%)$ a buena $(40,0 \%)$, y las enfermeras con un nivel moderado de satisfacción laboral presentan en un $75,0 \%$ calidad de vida regular y las enfermeras con nivel bajo de satisfacción laboral en un 63,0\% presentan un nivel regular de calidad de vida profesional. Ante la aplicación de la prueba estadística de independencia de criterios $(\mathrm{x} 2)$ se mostró $(\mathrm{p}=0,0013<0,05)$, determinando que sí existe una relación significativa entre el nivel de satisfacción laboral y el de calidad de vida profesional. 


\section{DISCUSIÓN}

En la tabla 01, los resultados evidencian que las dimensiones con mayor frecuencia en el nivel alto de satisfacción laboral en el profesional de enfermería son las dimensiones de satisfacción en el trabajo y competencia profesional; las dimensiones con un nivel bajo de satisfacción laboral en un porcentaje significativo son las dimensiones de promoción profesional, presión de trabajo y tensión relacionada en el trabajo; sin embargo, es notable observar que en casi todas las dimensiones más del 50\% de enfermeras presentan un nivel regular de satisfacción laboral.

Las dimensiones competencia profesional y satisfacción del trabajo son las predictorias a una alta satisfacción laboral, debido a que existe una compatibilidad entre la personalidad de la persona y el puesto de trabajo que este desempeñe. ${ }^{10}$ Por ello, los trabajadores prefieren trabajos que les den oportunidad de usar sus habilidades, conocimientos, aptitudes, actitudes y buen juicio asociados a su profesión, para poder desempeñarla de manera eficaz en todas las situaciones que corresponden al campo de su práctica". ${ }^{11}$ Es por eso, que el enriquecimiento del puesto a través de la expansión vertical del mismo puede elevar la satisfacción laboral, ya que se incrementa la libertad, independencia, variedad de tareas.

Adicionalmente, las dimensiones bajo el control del individuo (motivadores intrínsecos), como la competencia profesional y la satisfacción del trabajo, han proporcionado a las enfermeras un nivel alto de satisfacción laboral y las dimensiones que refieren a las condiciones en que se encuentra el individuo en relación con la empresa (extrínsecos), como la promoción profesional, la presión de trabajo, han generado un nivel bajo de satisfacción laboral, afirmando la teoría de Herzberg.

En la tabla 02, las dimensiones con un nivel bueno en la calidad de vida profesional son representadas sólo por dos dimensiones: la capacidad de realizar el trabajo y motivación intrínseca, a diferencia que las enfermeras con nivel malo de calidad de vida profesional presentan una mayor frecuencia en la dimensión carga de trabajo percibido (54,0\%); sin embargo, en la dimensión de recursos ligados al lugar de trabajo el $73,0 \%$ de las enfermeras presenta un nivel regular.

Los resultados permiten inferir que entre la calidad de vida profesional y la satisfacción laboral existe un patrón de relación, debido a que las dimensiones con una buena calidad de vida profesional son aquellas que tienen una connota- 
ción psicológica; por ende, la motivación intrínseca y los recursos ligados al trabajo son características que controla el individuo, como ganas de ser creativo, orgullo por su trabajo, motivación, satisfacción laboral, carga de responsabilidad, autonomía o libertad de decisión. Los ítems antes mencionados contemplan aspectos que potencian la calidad de vida profesional y que están más relacionados con diferencias individuales, ${ }^{12}$ que con aspectos de responsabilidad directa de la organización. Sin embargo, la percepción favorable en dichos factores es consecuencia de la función o valor social que tiene el trabajo para el individuo y del ajuste persona-cargo y también de la integración entre valores personales y organizacionales.

Según los resultados, las enfermeras muestran mayor importancia a la calidad de vida profesional psicológica o necesidades de orden superior conforme a Maslow ${ }^{2}$. Esto evidencia que la dimensión motivación intrínseca, capacidad de realizar el trabajo y recursos ligados al lugar del trabajo es percibida como jerárquicamente superior a las condiciones del entorno, en la valoración de la calidad de vida profesional.

Las enfermeras con un nivel bajo en las dimensiones de la calidad de vida profesional están relacionadas a los ítems carga de trabajo, estrés, prisas y agobios, presión recibida para realizar el trabajo, presión recibida para mantener el trabajo.

De acuerdo a los resultados en la tabla 03 , existe una relación significativa entre el nivel de satisfacción laboral y el nivel de calidad de vida profesional de las enfermeras del Hospital Regional Docente de Trujillo.

Con los resultados de la investigación se infiere que las condiciones laborales del profesional de enfermería están deteriorando la satisfacción laboral y, por tanto, calidad de vida profesional. Cada vez se obtienen más argumentos que soportan la premisa de que existe una expropiación de la esencia y del sujeto de cuidado que está llevando a desvirtuar la esencia del rol profesional debido a las condiciones de este profesional, eje de la salud.

\section{CONCLUSIONES}

Las dimensiones con un nivel alto de satisfacción laboral son las relacionadas con la competencia profesional y la propia satisfacción del trabajo y con un nivel bajo, la relacionadas con promoción profesional, presión en el trabajo; las dimensiones con un nivel bueno de calidad de vida profesional son la motivación intrínseca y la capacidad de realizar el trabajo y las de bajo nivel, la carga de trabajo percibido. 
Existe relación significativa entre la satisfacción laboral y la calidad de vida profesional.

\section{REFERENCIAS BIBLIOGRÁFICAS}

1 Fernández B, PARAViC T. Nivel de satisfacción laboral en enfermeras de hospitales públicos y privados de la provincia de Concepción, Chile. Cienc. enferm. [revista en la Internet]. 2003 Dic [citado 2011 Nov 01]; 9(2): 57-66. Disponible en: http://www.scielo.cl/scielo.php?script $=$ sci arttext\&pid $=$ S071795532003000200006\&lng $=$ es. oi:10.4067/S0717-95532003000200006.

2 NAVARRo A. El ejercicio profesional y la responsabilidad penal, civil, administrativa y ética disciplinaria derivada de su ejercicio. Rev. cienc. adm. financ. segur. soc [revista en la Internet]. 2006 [citado 2010 Feb 01]; 14(1): 11-54. Disponible en: http://www.scielo.sa.cr/scielo.php? script $=$ sci_arttext\&pid $=$ S1409-12592006000100002\&lng $=$ es.

3 Malvárez, M. 2005. Panorama de la fuerza de trabajo en enfermería en América Latina. Washington, D.C: OPS, http://www.ops.org.bo/textocompleto/ift26346.pdf

4 Ministerio DE SALud. Normas Legales. Accesado diciembre 2009. Disponible en:http//minsa. gob.pe/portada/transparencia/default2.asp.

5 HERZBERg F. 1966. Work and the nature of man, Cleveland Ohio: The world publishing company (Obra original).

6 MASLOw, A. 1954. Motivation Personality. Nueva York: Harper \& Row.

7 NúÑEZ GoNZÁLEZ E et al. 2007. Una propuesta destinada a complementar el cuestionario FontRoja de satisfacción laboral. Gac Sanit. 21(2):136-41.

8 SÁnchez, R, Álvarez, R, Lorenzo, S. 2003. Calidad de vida profesional de los trabajadores de atención primaria del área 10 de Madrid. Medifam. 13(4): 291-296.

9 Denise F. y Polit, D. 1990. Investigación científica en ciencias de la salud. México: McGrawHill Interamericana;

10 REIG, F. Y JAULI. 2003. Los recursos humanos en las organizaciones orientadas a la eficacia y al aprendizaje. Madrid: Editorial Thomson.

11 BLAY P. 1995. Evaluación de la competencia profesional ¿están cambiando los tiempos? España: Editorial Revista Atención Primaria.

12 GonZÁlez, P., Peiró, J.M. y Bravo, M.J. 1996. Calidad de vida laboral. En Peiró J. M. y Prieto, F.(Eds.). Tratado de Psicología del Trabajo: Aspectos psicosociales del trabajo. Vol. (2). Madrid: Síntesis.

\section{Dirección del autor}

Mg. Asunción Marcela Urbina Baltodano.

Filadelfia 266, Urbanización Santa Isabel.

Ciudad de Trujillo. 